\title{
RESUMEN
}

El objetivo de este articulo es determinar la relación entre la rentabilidad y el proceso logístico de las empresas exportadoras de derivados del Zinc- Callao. Motivado a que los procesos logísticos comprenden actividades de producción, comercialización y distribución de los bienes y servicios que una entidad promociona y estas pueden afectar la rentabilidad de una empresa al existir variación significativa en los costos ocultos que incurren en la optimización de la rentabilidad. La investigación se enfocó bajo el tipo de investigación cuantitativa, correlacional y de diseño no-experimental. Así mismo, de igual forma presentó, como muestra seis empresas del sector exportador de derivados del Zinc en el distrito del Callao. Finalmente en cuanto a la prueba de hipótesis general, se muestra que la magnitud del coeficiente cercana a uno $(r=0.947)$ y su signo positivo, indica una relación positiva, es decir cuando es mayor la percepción de que el proceso logístico se ejecuta de manera correcta, aumenta la rentabilidad, de las empresas exportadoras de productos derivados de zinc - Callao, lo que constituye un resultado esperado según el modelo teórico. Se concluye que existe una relación significativa positiva entre la rentabilidad y el proceso logístico de las empresas exportadoras de derivados del Zinc- Callao.

Palabras Claves: Proceso logístico, actividades productivas, rentabilidad.

\section{EL PROCESO LOGÍSTICO Y LA RENTABILIDAD EN LAS EMPRESAS EXPORTADORAS DE PRODUCTOS DERIVADOS DE ZINC - CALLAO}

\author{
Fernando Ostolaza Neyra'
}

Fechas de recepción: 17 de septiembre de 2020

Fecha de aceptación: 20 de octubre de 2020

DOI: https://doi.org/10.22370/riace.2020.9.1.2601

1 Corresponding Author. Universidad Nacional Mayor de San Marcos, Lima, Perú. fostolazan@gmail.com 


\title{
THE LOGISTIC PROCESS AND PROFIT IN EXPORTING COMPANIES OF PRODUCTS DERIVED FROM ZINC - CALLAO
}

\begin{abstract}
Objective: To determine the relationship between profitability and the logistics process of companies that export Zinc-Callao derivatives. Motivated that the logistics processes include activities of production, marketing and distribution of the goods and services that an entity promotes and these can affect the profitability of a company as there is significant variation in the hidden costs incurred in optimizing profitability. Method: The research was focused under the type of quantitative, correlational and non-experimental design research. Likewise, he also presented, as shown, six companies in the export sector of zinc derivatives in the Callao district. Results: Finally, regarding the general hypothesis test, it is shown that the magnitude of the coefficient close to one $(r=0.947)$ and its positive sign indicate a positive relationship, that is, when the perception that the logistics process is greater is greater. Correctly executed, increases the profitability of the exporting companies of zinc derivative products - Callao, which constitutes an expected result according to the theoretical model. Conclusions: It is concluded that there is a significant positive relationship between profitability and the logistics process of exporting companies of Zinc-Callao derivatives.
\end{abstract}

Keywords: Logistic process, productive activities, profitability. 


\section{INTRODUCCIÓN}

Los procesos logísticos son el conjunto de actividades destinadas a la organización de las tareas de producción, comercialización y distribución de los bienes y servicios que una entidad promociona. Por tanto, tratan de buscar la mejor solución en la optimización de los procedimientos que los integran, considerando cómo el mercado usa estos productos. Cuando una empresa coordina exitosamente estos procesos, sus actividades serán más efectivas y eficientes para cumplir con la demanda de los clientes y posicionarse en el mercado (Bass, 2018).

De igual modo, estos procesos se vinculan con la gestión del flujo de los servicios y bienes, tomando en consideración desde la compra de los materiales directos e indirectos, en su origen hasta la entrega del producto final. De tal modo, todas aquellas acciones donde se incluya el recorrido de materia prima e insumos que conforman los procesos logísticos, al igual que toda actividad que brinda un soporte apropiado para que se transformen dichos elementos en bienes terminados para el consumidor final, el almacenamiento y las compras (Monterroso, 2000).

Sin embargo, estos procesos logísticos al no tener un control adecuado, pueden generar fallas en la planificación por no fijar tiempos y flujos en las cadenas de suministros, lo que conlleva a planificar en base a información errónea, demoras de traslados de mercancías, debido a negligencias por parte de funcionarios en traslados marítimos, aéreos u otros, como también de los colaboradores de la entidad, pudiendo incurrir en inexactitud de los inventarios de la mercancía almacenada.

En tal sentido, diversos estudios de investigación exponen la asociación entre la rentabilidad y el proceso logístico de una empresa. Cabe mencionar a Julca \& Pretell (2017) realizaron un trabajo que se enfocó en realizar una comprobación sobre la aplicación de un diseño de gestión logística, la cual obtendrá una ventaja competitiva a la ferretería "El Ingeniero" E.I.R.L, en el sector construcción del distrito de Trujillo - 2015. En el estudio se concluyó que se logró establecer el perfil de base de las organizaciones que funcionan como ferreteras en el sector de la construcción. Asimismo, se elaboró un diseño de gestión logística que tenía como objetivo conservar el orden. En la primera fase se consideró la recopilación de datos mediante la entrevista y encuesta a los gerentes de las empresas ferreteras de Trujillo con una muestra de 94 empresas de una población de 442 ferreterías. Los resultados han permitido diagnosticar el estado actual de las empresas ferreteras en el mercado de la construcción. En la segunda fase se estructuró un diseño de gestión logística como propuesta específica que busca que todas las áreas de la empresa sean trabajadas de manera ordenada y organizada. Finalmente, en la tercera fase se considera la aplicación de la propuesta del diseño de gestión logística y su impacto significativo en lograr una ventaja competitiva en el mercado de la construcción en servicio y tiempo. El uso de los indicadores de la gestión logística responde al modelo de diseño de un sistema logístico, el cuál ayuda a mejorar el tiempo de respuesta en la atención al cliente; además de tener un 
inventario acorde con la demanda del mercado y satisfacer las necesidades del cliente. Así mismo permitirá mejorar la administración de los costos logísticos el cual logrará que el ahorro sea sostenido a partir de la ejecución. También se ha concluido que la implementación de un sistema de gestión logística, son capaces de generar ventaja competitiva y mejores beneficios en todos sus niveles.

Jara (2017), efectuó una investigación que se enfocó en la búsqueda de una solución para la disminución de los costos logísticos en el I.E.P "Liceo Mi Dulce Angelito" Callao-2017". Como resultados se obtuvo que la disminución de los costos provenientes de la gestión logística fueron $25.76 \%$, el cual está representado por un total de 3,123.27 \$ (S/ 11,243.76). Asimismo, se concluyó que la organización industrial en estudio logró una considerable reducción en los costos logísticos motivado a la implementación de la gestión en la logística de la empresa. Los resultados de la prueba de hipótesis principal y específicas de la investigación se aceptan por lo cual son válidas. El trabajo tuvo como conclusión que la empresa en estudio aplicando la Gestión Logística obtuvo resultados favorables en la reducción de los costos logísticos, esto mediante un trabajo de aplicación favorable y por la experiencia que se pueda emplear al trabajo.

Torres (2016), presenta la investigación a fin de estipular los costos de exportación y establecer su incidencia en la rentabilidad de la empresa Café del Norte S.A.C. Como resultados obtuvo que el análisis financiero, basado en un enfoque de costos de exportación y la organización industrial, tuvo un sobresaliente manejo de sus recursos, en comparación con los costos resultantes de los esfuerzos para generar ingresos que mejorará implícitamente la rentabilidad. Además, se contrastó el último ejercicio con los ejercicios de años anteriores y se obtuvo para el año 2013 un 11.5\% más de ingresos y para el 2014 un12.60\% más de ingresos con respecto a la sistematización y estructura organizada en la logística, obteniendo en la simulación 28,6\% de ingresos netos.

Yapias (2014) desarrolla su tesis con el fin de establecer en el cómo los factores internos y externos influyen directamente sobre la rentabilidad de las Empresas Agroindustriales Exportadoras de Maca de la Provincia de Junín, en Universidad Nacional del Centro del Perú. En este este mismo sentido, como resultados se planteó lo siguiente: los componentes internos y externos, a través de sus indicadores de gestión influyen directamente sobre la rentabilidad de las organizaciones industriales que se dedican a la exportación de maca. Según el análisis y evaluación de las variables en estudio, así como lo prueba de Chi cuadrada. Como conclusión se resalta que los factores antes mencionados influyen negativamente en la rentabilidad de las empresas que se dedican a la exportación de maca en la provincia de Junín.

Maier (2017), realizó un estudio enfocado en la gestión del área Logística en el proceso de control de una planta automotriz en Argentina. El mismo contó con una estructura que comienza con una explicación teórica del área a analizar, finalizando con alternativas de solución al problema planteado (desarrollo de la herramienta 
por parte del equipo de TI de la planta automotriz) con una metodología de estructura no experimental, un tipo de investigación descriptiva y correlacional, siendo de campo, directa y horizontal. Asimismo, se elaboró un análisis costo beneficio, lo que permitió obtener como resultado una tabla de control de gestión, a fin de establecer los ahorros, que rodeaba los 3 millones de USD en el tercer año de implementación, utilizando un instrumento de gestión eficiente quedando demostrado que, al utilizar una herramienta de gestión eficiente, se logró una mejora considerable en los resultados del trabajo del equipo de Logística de entrada. Además de corroborar que es un proyecto viable desde el punto de vista económico-financiero.

Bosque (2016), realizó un estudio para rediseñar el control interno del departamento de Stock y Compras de Inventarios, de la organización Centro Motor S.A. Se apoyó en un análisis de los circuitos diversos, visualizándose las falencias puntuales y las fallas individuales de estos, a través un análisis de la realidad económica de la compañía. El resultado obtenido se presenta, en la propuesta de aplicación, en la cual, en los procesos, se logró mejorar, optimizando la rentabilidad y así disponer de confiabilidad en la información para llevar a cabo una toma de decisiones eficaz.

Molina (2015), realizó un estudio para planear y aplicar un patrón logístico para mejorar en la compañía la distribución de productos publicitarios; asimismo, se comprobó la ausencia de una logística modelo. En consecuencia, la planificación en los procesos de compra estuvo ausente, así como el almacenamiento y recepción, las cuales se ejecutan con falta de coordinación y comunicación de las diversas áreas en post venta, aunado a esto el desconocimiento de los costos de la ruta de transporte en la distribución de los productos publicitarios al domicilio de los clientes, siendo el atraso que manifiesta el comprador llegando a alcanzar hasta 5 días. En la evaluación se obtuvo una Tasa interna de retorno del $46.81 \%$, con un valor actual neto de USD 32,389.64, obteniendo en años y 3 meses la recuperación del capital invertido, y un 2.02 de costo beneficio de que comprobaron que la propuesta es factible.

González (2015), presenta una investigación para establecer la factibilidad financiera de la comercialización y exportación de flores cortadas a partir de la provincia del Pichincha específicamente del cantón Cayambe, hasta Alemania en la ciudad de Hamburgo utilizando como metodología de estudio una investigación documental y de campo de nivel descriptivo. Como resultado se mencionó que: la aplicación de una nueva hectárea para la producción de flores presenta un costo de USD 138,575.00. Destinando solamente el 30\% de la producción a Alemania, el proyecto presenta un valor actual neto de $31,049.02 \$$; comprobando que es viable el proyecto de exportación involucrando los costos de los procesos logísticos.

Sintetizando, las conclusiones de los diferentes estudios de investigación de pregrado contenidos en los antecedentes de la investigación, los procesos logísticos comprenden actividades de producción, comercialización y distribución de los bienes y servicios que una entidad promociona y estas pueden afectar la 
rentabilidad de una empresa. De modo que el presente estudio espera determinar la relación entre la rentabilidad y el proceso logístico de las empresas exportadoras de derivados del Zinc- Callao.

\section{MATERIAL Y MÉTODOS}

La investigación se enfocó en un estudio cuantitativo, correlacional y de diseño no experimental. En este sentido, la población estuvo constituida por el total de empresas exportadoras de derivados del Zinc ubicadas en el distrito del Callao, las cuales estuvieron activas comercialmente en el año 2019, dicha población asciende a 6 empresas. Asimismo, el tamaño de la muestra fue de 36 colaboradores del área gerencial, administrativa y contable de 6 empresas exportadoras de productos derivados de zinc - Callao, mediante un muestreo no probabilístico. Los colaboradores poseían el cargo de gerente, administrador 0 asistente administrativo. Además, como variable independiente se consideró Proceso logístico y como variable dependiente Rentabilidad. En tal sentido, se postuló como hipótesis general que existe relación entre el proceso logístico y la rentabilidad de las empresas exportadoras de derivados del Zinc- Callao.

\section{RESULTADOS}

\section{Factores relacionados con el proceso logístico del almacenaje}

Los resultados de los ítems correspondientes a los factores relacionados con proceso logístico del almacenaje se plasman en la tabla 1, los cuales se tomaron de una muestra de 36 colaboradores del área gerencial, administrativa y contable de 6 empresas exportadoras de productos derivados de zinc, con un nivel de confianza del instrumento de coeficiente alfa de Cronbach igual a 0.972. Al respecto los hallazgos revelaron que la mayoría de los colaboradores afirman estar de acuerdo (38.9\%) y totalmente de acuerdo (11.1\%), con el hecho que en las empresas exportadoras de productos derivados de zinc - Callao, la cantidad mensual de derivados de Zinc que mantiene almacenada la empresa, responde a su expectativa de demanda, sin embargo, una cuarta parte de la muestra estuvo totalmente en desacuerdo. De igual forma, la mayoría de la muestra afirmó estar de acuerdo (27.8\%) o totalmente de acuerdo (19.4\%) con el hecho que la cantidad de derivados de Zinc que se dirige al almacén, se corresponde con las órdenes de compra recibidas por las empresas exportadoras de productos derivados de zinc - Callao. Asimismo, la mayoría opinó estar de acuerdo (30.6\%) o totalmente de acuerdo (16.7\%), en que las cantidades almacenadas son suficientes para atender el pedido de un cliente. Finalmente, también están de acuerdo (30.6\%) y totalmente de acuerdo (18.1\%), en que pocas veces se presentan discrepancia entre las cantidades despachadas desde el almacén y las indicadas en las órdenes de compra. En resumen, entre los colaboradores encuestados hay una tendencia 
agregada positiva en cuanto a los factores relacionados con el proceso logístico del almacenaje.

Tabla 1. Ítems referidos a los factores relacionados con el proceso logístico del almacenaje

\begin{tabular}{|c|c|c|c|c|c|c|c|c|c|}
\hline \multirow{2}{*}{\multicolumn{2}{|c|}{ Núm. Ítem }} & \multicolumn{2}{|r|}{ TD } & ED & $\mathbf{N}$ & \multicolumn{2}{|c|}{ DA } & \multicolumn{2}{|r|}{ TA } \\
\hline & & $\mathbf{n}$ & $\%$ & $\mathrm{n}$ & N $\%$ & $\mathbf{N}$ & $\%$ & $\mathbf{n}$ & $\%$ \\
\hline 1 & $\begin{array}{l}\text { La cantidad mensual de derivados } \\
\text { de Zinc que mantiene almacenada la } \\
\text { empresa, responde a su expectativa } \\
\text { de demanda }\end{array}$ & 9 & $925.0 \%$ & $411.1 \%$ & $513.9 \%$ & & $38.9 \%$ & & $411.1 \%$ \\
\hline 2 & $\begin{array}{l}\text { La cantidad de derivados de Zinc que } \\
\text { se dirige al almacén, se corresponde } \\
\text { con las órdenes de compra recibidas }\end{array}$ & 6 & 6 16.7\% & $616.7 \%$ & $719.4 \%$ & & $27.8 \%$ & 7 & $7 \quad 19.4 \%$ \\
\hline 3 & $\begin{array}{l}\text { Las cantidades almacenadas son } \\
\text { suficientes para atender el pedido de } \\
\text { un cliente }\end{array}$ & 6 & 6 16.7\% & $513.9 \%$ & $822.2 \%$ & & $30.6 \%$ & 6 & $6 \quad 16.7 \%$ \\
\hline 4 & $\begin{array}{l}\text { Pocas veces se presentan } \\
\text { discrepancia entre las cantidades } \\
\text { despachadas desde el almacén y las } \\
\text { indicadas en las órdenes de compra }\end{array}$ & 2 & $2 \quad 5.6 \%$ & $822.2 \%$ & $822.2 \%$ & & $25.0 \%$ & 9 & $925.0 \%$ \\
\hline & $\begin{array}{l}\text { Total, factores relacionados con el } \\
\text { proceso logístico del almacenaje }\end{array}$ & 23 & $16.0 \%$ & $2316.0 \%$ & $2819.4 \%$ & 44 & $30.6 \%$ & 26 & $18.1 \%$ \\
\hline
\end{tabular}

Fuente: elaboración propia

\section{Factores de transporte}

Al examinar los resultados correspondientes a los ítems de la variable proceso logístico en su dimensión de factores de transporte se muestra una tendencia de respuestas en acuerdo a los ítems, la cual se plasma en la tabla 2. En este sentido, la evidencia muestral revela que la mayoría de los colaboradores opinan estar de acuerdo (38.9\%) y totalmente de acuerdo (11.1\%), con el hecho que en el seno de las empresas exportadoras de productos derivados de zinc - Callao, el producto es transportado en contenedores con la unitarización adecuada, no obstante, un $22 \%$ de la muestra, mostró su desacuerdo al respecto. De igual forma, la mayoría de los colaboradores afirmaron estar de acuerdo (44.4\%) o totalmente de acuerdo (11.1\%), con el hecho los costos de transporte al puerto no se han incrementado en el último periodo fiscal. Igualmente, una gran parte manifestó estar en desacuerdo (22.2\%) o totalmente en desacuerdo (16.7\%), con la afirmación que los costos de carga del buque no se han incrementado en el último periodo fiscal. Finalmente, también están de acuerdo (38.2\%) y totalmente de acuerdo (13.9\%), en que los costos administrativos se han incrementado en el último periodo fiscal. En resumen, entre los colaboradores encuestados, hay una tendencia de acciones en pro de obtener a su favor los factores de la industria, con debilidades en el conocimiento del cliente y de la demanda internacional. 
Tabla 2. Ítems referidos a los factores de transporte

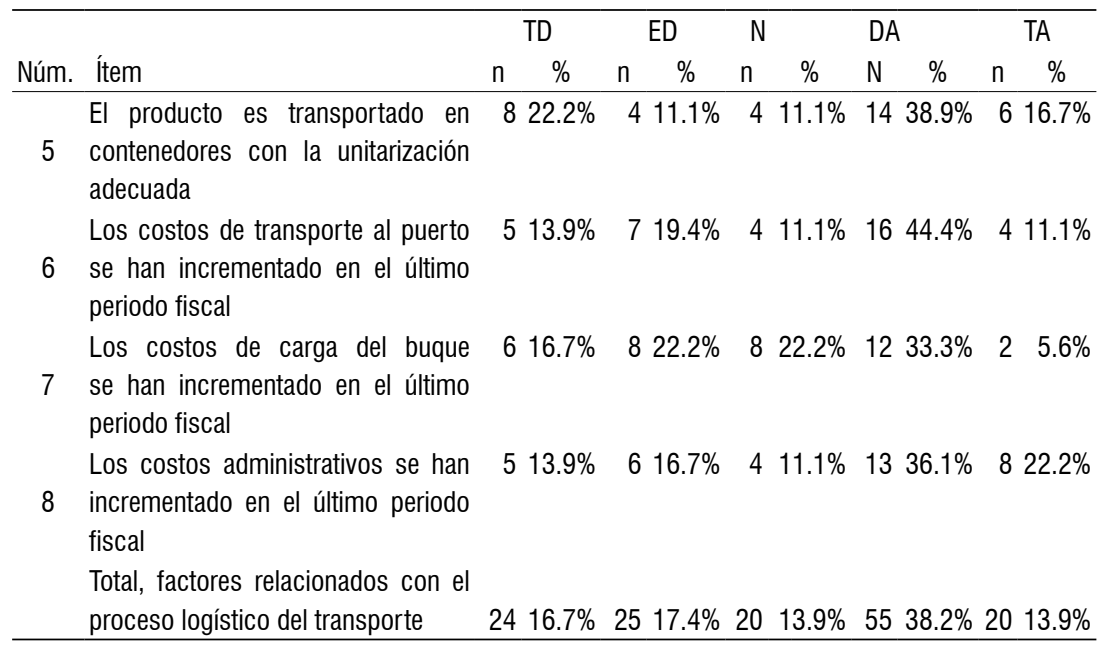

Fuente: Elaboración propia

\section{Factores de tiempo de ejecución logística de las empresas exportadoras de productos derivados de zinc - Callao}

Factores de tiempo de ejecución logística, son todos aquellos elementos que desde el seno de los procesos operativos de empresas exportadoras de productos derivados de zinc - Callao afectan el tiempo de ejecución de su proceso logístico. En este sentido, la tabla 3 nos resumen los ítems correspondientes a dicha dimensión. Se evidencia que la mayoría de la muestra afirma estar de acuerdo (36.1\%) y totalmente de acuerdo (22.2\%), con que las empresas exportadoras de productos derivados de zinc - Callao La movilización de las cargas hasta los buques se realiza en el tiempo previsto, no obstante, un $22.2 \%$ se encuentra en desacuerdo con dicho ítem. En un sentido similar, la mayoría de los colaboradores señalaron estar de acuerdo (36.1\%) o totalmente de acuerdo (16.7\%), con la afirmación que la descarga del buque se realiza en el tiempo previsto y sin contratiempos. Igualmente, una parte mayoritaria manifestó estar en desacuerdo (22.2\%) o totalmente en desacuerdo (16.7\%), con la afirmación que las empresas exportadoras de productos derivados de zinc - Callao La empresa realiza sus entregas en el tiempo previsto y acordado con el comprador. En último lugar, también están de acuerdo (33.3\%) y totalmente de acuerdo (8.3\%), en La empresa de transporte realiza sus trámites legales establecidos (sanitarias, de carga, etc.) en el tiempo previsto, sin embargo, otra parte de la muestra se manifestó en desacuerdo (25\%). En resumen, se puede decir que en forma agregada entre los colaboradores encuestados hay una tendencia de acciones en pro de Factores de tiempo de ejecución logística de las empresas exportadoras de productos derivados de zinc - Callao. 
Tabla 3. Ítems referidos a los factores de tiempo de ejecución logística de las empresas exportadoras de productos derivados de zinc - Callao

\begin{tabular}{|c|c|c|c|c|c|c|c|c|c|c|c|}
\hline \multirow[b]{2}{*}{ Núm. } & \multirow[b]{2}{*}{ Ítem } & \multicolumn{2}{|r|}{ TD } & \multicolumn{2}{|r|}{ ED } & \multicolumn{2}{|r|}{$\mathrm{N}$} & \multicolumn{2}{|c|}{ DA } & \multicolumn{2}{|r|}{ TA } \\
\hline & & $n$ & $\%$ & $\mathrm{n}$ & $\%$ & $\mathrm{n}$ & $\%$ & $\mathbf{N}$ & $\%$ & $\mathbf{n}$ & $\%$ \\
\hline 9 & $\begin{array}{l}\text { La movilización de las cargas hasta } \\
\text { los buques se realiza en el tiempo } \\
\text { previsto }\end{array}$ & 3 & $8.3 \%$ & 8 & $22.2 \%$ & 4 & $11.1 \%$ & 13 & $36.1 \%$ & & $822.2 \%$ \\
\hline 10 & $\begin{array}{l}\text { La descarga del buque se realiza en el } \\
\text { tiempo previsto y sin contratiempos }\end{array}$ & 5 & $13.9 \%$ & 5 & $13.9 \%$ & & $19.4 \%$ & 13 & $36.1 \%$ & & $616.7 \%$ \\
\hline 11 & $\begin{array}{l}\text { La empresa realiza sus entregas en } \\
\text { el tiempo previsto y acordado con el } \\
\text { comprador }\end{array}$ & 8 & $22.2 \%$ & 6 & $16.7 \%$ & & $16.7 \%$ & 13 & $36.1 \%$ & & $\begin{array}{l}3.3 \% \\
\end{array}$ \\
\hline 12 & $\begin{array}{l}\text { La empresa de transporte realiza } \\
\text { sus trámites legales establecidos en } \\
\text { el tiempo previsto, (sanitarias, de } \\
\text { carga, etc.) }\end{array}$ & 5 & $13.9 \%$ & 9 & $25.0 \%$ & & $19.4 \%$ & 12 & $33.3 \%$ & & $3 \quad 8.3 \%$ \\
\hline & $\begin{array}{l}\text { Factores de tiempo de ejecución } \\
\text { logística de las empresas } \\
\text { exportadoras de productos derivados }\end{array}$ & & & & & & & & & & \\
\hline & de zinc - Callao & 21 & $14.6 \%$ & 28 & $19.4 \% 2$ & & $16.7 \%$ & 51 & $35.4 \%$ & & $013.9 \%$ \\
\hline
\end{tabular}

Fuente: elaboración propia

\section{Nivel de proceso logístico}

Al agregar los puntajes de cada ítem se logró estimar el nivel de proceso logístico de la muestra de empresas exportadoras de productos derivados de zinc - Callao. En este sentido, se obtuvo que el $50 \%$ de la muestra se ubicó en un nivel de proceso logístico alto, seguido de un $25 \%$ con un nivel medio un $25 \%$ con un nivel bajo.

Tabla 4. Nivel de proceso logístico

\begin{tabular}{lllll}
\hline Escala [puntos] & Frecuencia & Porcentaje & $\begin{array}{l}\text { Porcentaje } \\
\text { válido }\end{array}$ & $\begin{array}{l}\text { Porcentaje } \\
\text { acumulado }\end{array}$ \\
\hline Bajo [0-20] & 9 & 25.0 & 25.0 & 25.0 \\
Medio [21-40] & 9 & 25.0 & 25.0 & 50.0 \\
Alto [41-60] & 18 & 50.0 & 50.0 & 100.0 \\
Total & 36 & 100.0 & 100.0 & \\
\hline
\end{tabular}

Fuente: elaboración propia 


\section{Rentabilidad}

\section{Rentabilidad en relación con las ventas}

La primera dimensión que aproxima la realidad de la rentabilidad en las organizaciones industriales exportadoras de productos derivados de zinc Callao es la rentabilidad en relación con las ventas, la cual se resume en la tabla 5. Al respecto coinciden en estar de acuerdo o muy de acuerdo con elementos como: la disminución de costos de producción en este periodo (30.6\% y 19,4\% respectivamente), producción en niveles de eficiencia operativa (30.6\% y 13.9\%) y producción en un alto nivel (38.9\% y $5.6 \%)$. En resumen, la mayoría de los colaboradores afirman estar al menos de acuerdo con los ítems relacionados a la gestión de la rentabilidad con relación a las ventas, con debilidades en el aumento de las ventas y en el nivel de precios.

Tabla 5. Ítems referidos a la rentabilidad en relación con las ventas

\begin{tabular}{|c|c|c|c|c|c|c|c|c|c|}
\hline \multirow{2}{*}{\multicolumn{2}{|c|}{ Núm. Ítem }} & \multicolumn{2}{|r|}{ TD } & ED & N & \multicolumn{2}{|c|}{ DA } & \multicolumn{2}{|r|}{ TA } \\
\hline & & $\mathrm{n}$ & $\%$ & $\%$ & $\%$ & $\mathrm{~N}$ & $\%$ & $\mathrm{n}$ & $\%$ \\
\hline 1 & $\begin{array}{l}\text { Se han disminuido los costos de } \\
\text { producción en este período. }\end{array}$ & & $19.4 \%$ & $719.4 \%$ & $411.1 \%$ & 11 & $30.6 \%$ & & $19.4 \%$ \\
\hline 2 & $\begin{array}{l}\text { Se produce con niveles de eficiencia } \\
\text { operativa. }\end{array}$ & 7 & $19.4 \%$ & $616.7 \%$ & $719.4 \%$ & 11 & $30.6 \%$ & 5 & $13.9 \%$ \\
\hline 3 & $\begin{array}{l}\text { Presentan volúmenes de producción } \\
\text { altos. }\end{array}$ & & $11.1 \%$ & $719.4 \%$ & $925.0 \%$ & 14 & $38.9 \%$ & 2 & $5.6 \%$ \\
\hline 4 & $\begin{array}{l}\text { Se han aumentado los precios de los } \\
\text { productos durante este período. }\end{array}$ & 10 & $27.8 \%$ & $38.3 \%$ & $616.7 \%$ & 13 & $36.1 \%$ & 4 & $11.1 \%$ \\
\hline 5 & $\begin{array}{l}\text { Los niveles de ventas presentan una } \\
\text { tendencia al alza. }\end{array}$ & 5 & $13.9 \%$ & $822.2 \%$ & $411.1 \%$ & & $25.0 \%$ & 10 & $27.8 \%$ \\
\hline 6 & $\begin{array}{l}\text { Los gastos de la empresa se han } \\
\text { mantenido estables. }\end{array}$ & 6 & $16.7 \%$ & $719.4 \%$ & $513.9 \%$ & 10 & $27.8 \%$ & 8 & $22.2 \%$ \\
\hline 7 & $\begin{array}{l}\text { Se tiene una eficiente administración } \\
\text { fiscal. }\end{array}$ & 7 & $19.4 \%$ & $513.9 \%$ & $822.2 \%$ & 7 & $19.4 \%$ & 9 & $25.0 \%$ \\
\hline 8 & $\begin{array}{l}\text { Se cumplen con los requerimientos } \\
\text { tributarios. }\end{array}$ & 6 & $16.7 \%$ & 7 19.4\% & $411.1 \%$ & 11 & $30.6 \%$ & 8 & $22.2 \%$ \\
\hline 9 & $\begin{array}{l}\text { Las obligaciones tributarias han } \\
\text { aumentado. }\end{array}$ & 3 & $8.3 \%$ & $1027.8 \%$ & $513.9 \%$ & 11 & $30.6 \%$ & 7 & $19.4 \%$ \\
\hline & $\begin{array}{l}\text { Rentabilidad en relación con las ventas } \\
\text { (ROV) }\end{array}$ & 55 & $17.0 \%$ & 6018.5 & $5216.0 \%$ & TI & $29.9 \%$ & 60 & $18.5 \%$ \\
\hline
\end{tabular}

Fuente: elaboración propia

\section{Rentabilidad en relación con la inversión}

Los ítems relacionados con la rentabilidad en relación con la inversión se resumen en la tabla 6. Los resultados muestran que los colaboradores parecen estar de acuerdo o muy de acuerdo, con la afirmación que en el seno de la empresas exportadoras de productos derivados de zinc - Callao se distribuye la utilidad 
generada en nuevas inversiones (22.2\% y $25 \%$ respectivamente), asimismo, se asigna la utilidad entre los socios (27.8\% y $19.4 \%$ respectivamente), de igual manera se establece en su gestión administrativa la relación que existe entre la inversión y la utilidad (27.8\% y $25 \%$ respectivamente). En resumen, el personal administrativo-contable de las empresas exportadoras de productos derivados de zinc - Callao está de acuerdo o muy de acuerdo en efectuar estrategias para aumentar la rentabilidad de la inversión, pero tienen debilidades en la gestión de los activos para generar niveles de utilidad.

Tabla 6. Rentabilidad en relación con la inversión

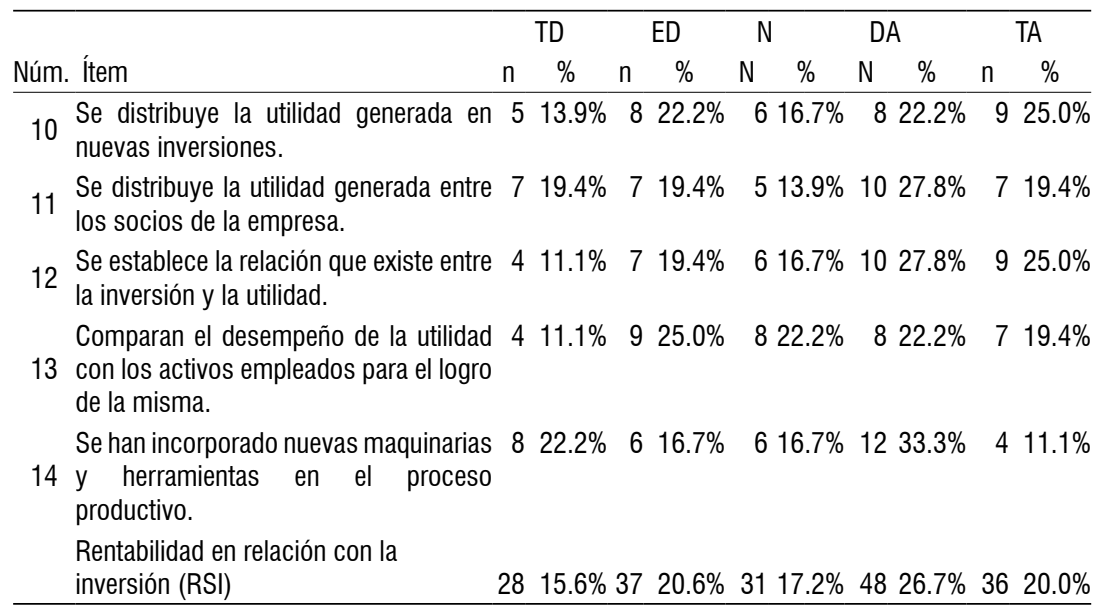

Fuente: elaboración propia.

\section{Rentabilidad en relación con el capital}

La rentabilidad en relación al capital, es la última dimensión evaluada y resumida en la tabla 7. Los resultados evidencian que los colaboradores parecen coincidir en cómo se relaciona la ganancia neta con el capital invertido para obtenerla (22.2\% y $30.6 \%$ respectivamente), poseer niveles de deuda bajos ( $25 \%$ y $16.7 \%$ ), gastos administrados de forma eficiente (22.2\% y $22.2 \%$ respectivamente). Por otra parte, existe la tendencia de afirmar estar de acuerdo o muy de acuerdo con el hecho que: comparan el desempeño de la utilidad con resultados históricos $(30.6 \%$ y $19.4 \%$ respectivamente). Asimismo, una parte de la muestra está totalmente en desacuerdo y en desacuerdo, con el hecho que la inversión realizada ha respondido a la necesidad de expansión en el mercado (11.1\% y $30.6 \%$ respectivamente). En resumen, la mayoría de los colaboradores afirman estar de acuerdo en la aplicación de estrategias en pro de obtener rentabilidad sobre el capital, con presencia de debilidades en el nivel de inversión según la expansión del mercado y la asunción de riesgos mínimos. 
Tabla 7. Ítems referidos a la rentabilidad en relación al capital

\begin{tabular}{|c|c|c|c|c|c|c|c|c|c|}
\hline \multirow{2}{*}{\multicolumn{2}{|c|}{ Núm. Ítem }} & \multicolumn{2}{|r|}{ TD } & \multicolumn{2}{|c|}{ ED } & \multicolumn{2}{|c|}{$\mathrm{N}$} & DA & TA \\
\hline & & $\mathrm{n}$ & $\%$ & $\mathrm{n}$ & $\%$ & $\mathrm{~N}$ & $\%$ & $\mathrm{~N}$ & $\%$ \\
\hline 15 & $\begin{array}{l}\text { Se relaciona la ganancia neta con el } \\
\text { capital invertido para obtenerla. }\end{array}$ & 5 & $13.9 \%$ & 5 & $13.9 \%$ & & $19.4 \%$ & $822.2 \%$ & $1130.6 \%$ \\
\hline 16 & Presentan niveles de deuda bajos. & 8 & $22.2 \%$ & 3 & $8.3 \%$ & & $27.8 \%$ & $925.0 \%$ & $616.7 \%$ \\
\hline 17 & $\begin{array}{l}\text { Los gastos se han administrados de } \\
\text { manera efectiva. }\end{array}$ & 4 & $11.1 \%$ & 7 & $19.4 \%$ & & $25.0 \%$ & $822.2 \%$ & $822.2 \%$ \\
\hline 18 & $\begin{array}{l}\text { Comparan el desempeño de la utilidad } \\
\text { con resultados históricos. }\end{array}$ & 5 & $13.9 \%$ & 6 & $16.7 \%$ & & $19.4 \%$ & $1130.6 \%$ & $719.4 \%$ \\
\hline 19 & $\begin{array}{l}\text { Comparan el desempeño de la utilidad } \\
\text { con otras empresas del mercado. }\end{array}$ & 6 & $16.7 \%$ & 5 & $13.9 \%$ & & $16.7 \%$ & $1233.3 \%$ & $719.4 \%$ \\
\hline 20 & $\begin{array}{l}\text { Se han aprovechado oportunidades de } \\
\text { inversión fuertes. }\end{array}$ & 7 & $19.4 \%$ & 5 & $13.9 \%$ & & $16.7 \%$ & $1130.6 \%$ & $719.4 \%$ \\
\hline 21 & $\begin{array}{l}\text { La inversión realizada ha respondido } \\
\text { a la necesidad de expansión en el } \\
\text { mercado. }\end{array}$ & 4 & $11.1 \%$ & 11 & $30.6 \%$ & & $22.2 \%$ & $822.2 \%$ & $513.9 \%$ \\
\hline 22 & Aceptan riesgos financieros & 5 & $13.9 \%$ & 9 & $25.0 \%$ & & $16.7 \%$ & $822.2 \%$ & $822.2 \%$ \\
\hline & Rental & 44 & 15.3 & 51 & 17.7 & & $.5 \%$ & $7526.0 \%$ & $20.5 \%$ \\
\hline
\end{tabular}

Fuente: elaboración propia

\section{Nivel de la rentabilidad}

Al agregar los puntos correspondientes a cada estrategia financiera para obtener rentabilidad, fue posible clasificar dicho total en tres categorías cualitativas. Los resultados indican que los colaboradores opinan que las acciones para conseguir la rentabilidad se ubican en un nivel medio 0 alto (38.9\% y $38.9 \%$ respectivamente) y muy pocos consideran que es bajo (22.2\%).

Tabla 8. Nivel de la rentabilidad

\begin{tabular}{lrrrr}
\hline Escala [puntos) & Frecuencia & Porcentaje & Porcentaje válido & $\begin{array}{c}\text { Porcentaje } \\
\text { acumulado }\end{array}$ \\
\hline Bajo [0 a 36] & 8 & 22.2 & 22.2 & 22.2 \\
Medio [37 a 74] & 14 & 38.9 & 38.9 & 61.1 \\
Alto [74 a 110] & 14 & 38.9 & 38.9 & 100.0 \\
Total & 36 & 100.0 & 100.0 & \\
\hline
\end{tabular}

Fuente: Elaboración propia 


\section{DISCUSIÓN}

En cuanto a la prueba de hipótesis general, se muestra que la magnitud del coeficiente cercana a uno ( $r=0.947)$ y su signo positivo, indica una relación positiva y significativa, es decir cuando es mayor la percepción de que el proceso logístico se ejecuta de manera correcta, aumenta la rentabilidad, de las organizaciones industriales que se dedican a la exportación de productos derivados de zinc Callao, lo que constituye un resultado esperado según el modelo teórico.

En el caso de la prueba de hipótesis número 1, la cual, es formulada de la siguiente manera: preexiste una relación significativa entre la rentabilidad y el proceso logístico, en relación a las ventas de las empresas exportadoras de derivados del Zinc- Callao. Al respecto coinciden en estar de acuerdo o muy de acuerdo con elementos como: la disminución de costos de producción en este periodo (30.6\% y $19,4 \%$ respectivamente), producción en niveles de eficiencia operativa (30.6\% y $13.9 \%$ ) y producción en un alto nivel (38.9\% y $5.6 \%)$, lo que conlleva a que la hipótesis ya mencionada muestra que la magnitud del coeficiente es cercana a uno $(r=0.932)$, y signo positivo, lo que sugiere una relación positiva y significativa, es decir, cuando mejora la percepción de que el proceso logístico se ejecuta de manera correcta, aumenta la rentabilidad sobre las ventas de las empresas exportadoras de productos derivados de zinc - Callao siendo estos resultado acordes con el modelo teórico. Por lo que se considera que preexiste una relación significativa entre la rentabilidad y el proceso logístico, en relación a las ventas.

En cuanto a la prueba de hipótesis número 2, la cual, es formulada de la siguiente forma: preexiste relación significativa entre la rentabilidad y el proceso logístico, en relación a inversión de las empresas que se dedican a la exportación de derivados del Zinc- Callao, muestran que los colaboradores de las empresas exportadoras de productos derivados de zinc - Callao están de acuerdo o muy de acuerdo en efectuar estrategias para aumentar la rentabilidad de la inversión, pero tienen debilidades en la gestión de los activos para generar niveles de utilidad. Como resultado se obtuvo que la magnitud del coeficiente es cercana a uno $(r=0.925)$, así como su signo positivo, sugiere una relación positiva y significativa, es decir, cuando mejora la percepción de que el proceso logístico se ejecuta de manera correcta, aumenta la rentabilidad sobre la inversión, siendo acorde al resultado esperado con el modelo teórico. Por lo que se considera que preexiste una relación significativa entre la rentabilidad y el proceso logístico, en relación a la inversión.

En el caso de la prueba de hipótesis número 3, formulada de esta forma: preexiste relación significativa entre la rentabilidad y el proceso logístico en relación al capital de las empresas exportadoras de derivados del Zinc- Callao, la cual, la mayoría de los colaboradores afirman estar de acuerdo en la aplicación de estrategias en pro de obtener rentabilidad sobre el capital, con presencia de debilidades en el nivel de inversión según la expansión del mercado y la asunción de riesgos mínimos. Se obtiene un resultado de magnitud del coeficiente cercana a uno $(r=0.855)$ y su signo positivo, muestra una relación positiva y fuerte, es decir, cuando mejora la 
percepción de que el proceso logístico se ejecuta de manera correcta se incrementa la rentabilidad sobre el capital, siendo acorde al resultado esperado según el modelo teórico. Por lo que se considera que preexiste una relación significativa entre la rentabilidad y el proceso logístico.

\section{REFERENCIAS}

BASS, B. (2018). ¿Qué son los procesos logísticos? Recuperado de: https:// pyme.lavoztx.com/qu-son-los-procesos-logsticos-5028.html

BOSQUE, R. (2016). El control interno y su incidencia en la rentabilidad de la empresa Centro Motor S.A. Instituto Universitario Aeronáutico. (Tesis de pregrado), Córdoba, Argentina.

GONZÁLEZ J (2015). Estudio financiero para determinar la factibilidad de la empresa Flores de la Victoria (Tesis pregrado). Universidad Politécnica Salesiana. Guayaquil-Ecuador.

JARA, C. (2017). Aplicación de la gestión logística para reducción de costos logísticos en el I.E.P - Liceo Mi Dulce Angelito Callao-2017 (Tesis de grado). Universidad Cesar Vallejo. Lima, Perú.

JULCA, I., \& PRETELL, A. (2017). Diseño de un sistema de gestión logística para generar ventaja competitiva de la ferretería "el ingeniero" E.I.R.L. en el sector construcción del distrito de Trujillo - 2015 (Tesis de grado). Universidad Privada Antenor Orrego. Trujillo, Perú.

MAIER, N. (2017). Implementación de un sistema de control logístico en el área de logística de entrada de una planta automotriz (Tesis de pregrado). Universidad Torcuato Di Tella, Buenos Aires, Argentina.

MOLINA, J. (2015). Planificación e implementación de un modelo logístico para optimizar la distribución de productos publicitarios en la empresa Letreros Universales S.A. (Tesis pregrado). Universidad Politécnica Salesiana. Guayaquil-Ecuador.

MONTERROSO, E. (2000). El proceso logístico y la gestión de la cadena de abastecimiento. Recuperado de: https://www.virtualpro.co/biblioteca/elproceso-logistico-y-la-gestion-de-la-cadena-de-abastecimiento

TORRES, H. (2016). Los costos de exportación y su incidencia en la rentabilidad de Café del Norte S.A.C la coipa en el periodo 2013 y 2014 (Tesis pregrado). Universidad Católica Santo Toribio de Mogrovejo. Cajamarca-Perú.

YAPIAS, A. (2014). Rentabilidad de las Empresas Agroindustriales Exportadoras de Maca de la Provincia de Junín (Tesis pregrado). Universidad Nacional Del Centro Del Perú. Junín, Perú. 